\title{
A combined experimental and computational study of a vertical gust generator in a wind tunnel
}

\author{
Naresh Poudel ${ }^{1}$, and Meilin $\mathrm{Yu}^{2}$ \\ University of Maryland, Baltimore County, Baltimore, MD, 21250, USA \\ Zachary F. Smith ${ }^{3}$ \\ University of Maryland, College Park, MD, 20742, USA \\ and \\ John T Hrynuk ${ }^{4}$ \\ US Army Research Lab, Aberdeen Proving Ground, MD, 21005, USA
}

\begin{abstract}
Experimental and numerical studies have been performed to investigate the performance of a vertical gust generator in a wind tunnel. Results from both experimental Particle Image Velocimetry (PIV) and those from numerical simulations of low-Mach-numberpreconditioned compressible Naiver-Stokes equations on unstructured grids using high-order spectral difference (SD) agreed reasonably well with each other. The gust was generated by ducting flow through the floor of the wind tunnel and at the wall of the numerical domain. The gust velocity profile bends downstream when interacting with freestream causing the incoming freestream flow to bend upward. The vertical gust changed the effective angle of attack over the airfoil when interacting with the freestream velocity. Due to the interaction of gust over the airfoil, the stationary airfoil at a nominal zero degree angle of attack undergo flow separation under stalled conditions.
\end{abstract}

\section{Nomenclature}

Re $\quad=$ Reynolds Number

$\mathrm{c} \quad=$ chord

$\Delta t \quad=$ time step

$\mathrm{t}^{*} \quad=$ convective time

$U_{\infty} \quad=$ freestream velocity

$V_{g} \quad=$ gust velocity

\section{Introduction}

The gust response of Micro Air Vehicles (MAVs) is a fundamental problem associated with the flight stability, maneuverability, and control due to the small size of the vehicles. Early in aviation history gust research focused primarily on the response of large aircraft ${ }^{1}$, but these tests seldom reflected the aerodynamic conditions experienced by MAVs. Unlike large aircraft operating at high forward speed, the slower velocity of MAVs increases their likelihood of experiencing natural vertical gusts larger in magnitude than their forward flight velocity. Current understanding of gusts is usually limited by the methods researchers have used to develop vertical gusts in an experimental setting. While CFD studies of gust interactions are not uncommon ${ }^{2-4}$, they are seldom compared to experimental results. This study undertakes a comparison of gust wing interaction simulated experimentally with a vertical gust generator in a wind tunnel environment and numerically with advanced unsteady CFD tools. Experimental results from Smith et al. ${ }^{5}$ are further analyzed and used to develop inlet and boundary conditions of a

\footnotetext{
1 Graduate Student, Department of Mechanical Engineering, and AIAA Student Member. Email: npoudel1@umbc.edu.

${ }^{2}$ Assistant Professor, Department of Mechanical Engineering, and AIAA Senior Member. Email: mlyu@umbc.edu.

${ }^{3}$ Graduate Research Assistant, Department of Aerospace Engineering, and AIAA Member.

${ }^{4}$ Mechanical Research Engineer, Vehicle Technology Directorate, US Army Research Lab.
} 
CFD model of the experimental setup. This CFD model will be used to further explore extremely time consuming experimental variables, such as turbulence reduction, and widen the understanding of mechanism of gust generation in a wind tunnel.

\section{Background}

Study of the fundamental response of MAV scale vehicles and subcomponents to vertical gusts is key to the development of active and passive gust rejection methods for future MAVs. Past studies usually focused on small amplitude transient vertical gusts, wherein a small change in wing angle of attack $(\alpha)$ was modeled as a transient. These methods include Wagner's indicial function and Kussner's function for estimating the lift changes as a function of gust encounter. However, recent work by $\mathrm{Smith}^{6}$ has shown that a vertical gust encounter in a wind tunnel environment bears little resemblance to the flow conditions of a static wing. This result suggested that linear theories are unlikely to ever properly represent gust encounters.

Other methods have previously been used for the study of vertical gust encounters. Traditionally a diffuse vortex generated upstream of test models was used to simulate vertical gust interactions (Patel and Hancock ${ }^{7}$, Buell ${ }^{8}$ ). While the generated vortex diffused before hitting the model, the method provides only transient up and downdraft behavior. Another common gust generating method is to actuate a wing under gust like maneuvers, i.e. plunging wings, in a water tunnel or tow tank (Baik et al. ${ }^{9}$; Perrotta et al. ${ }^{10}$ ). These methods have been shown to be effective for the study of gust interactions, but each has unique challenges. Plunging wings have high potential to mimic some gust like behaviors, but it remains unknown the extent of the differences between moving-body and moving-fluid gust interactions. Towing tank studies like Perrotta et al. ${ }^{10}$ produce high quality results but were limited in their transient nature, where the wing must keep moving and pass through the gust. This limited their ability to create a step-functionlike gust which would simulate a MAV flying into an updraft wider than the vehicle itself.

\section{Experimental Methods}

A vertical gust generating device was developed and connected to the low speed Microsystem Aeromechanics Wind Tunnel (MAWT) facility at the Army Research Lab. This wind tunnel has a $3 \times 3 \times 6 \mathrm{ft}$. test section and operates at speed from $1-25 \mathrm{~m} / \mathrm{s}$. The gust generator was designed as a parallel closed-loop, fan driven jet that entered the wind tunnel from the bottom, Fig. 1. The design of the gust generate was highly detailed in Smith et al. ${ }^{5}$ and Smith ${ }^{6}$. The gust generator was characterized using PIV for its effectiveness in changing the flow angle at a wing model location in the tunnel. The center of the gust inlet was placed $13 \mathrm{~cm}$ ahead of the $1 / 4$ chord location of a NACA 0012 full span model which was used in testing in Smith and Smith et al. The wind tunnel was run at $1.5 \mathrm{~m} / \mathrm{s}$ and neglecting any change in free stream associated with the gust, the chord Reynolds number associated with the experiments was $\mathrm{Re}_{\mathrm{c}}$ $=12,000$.

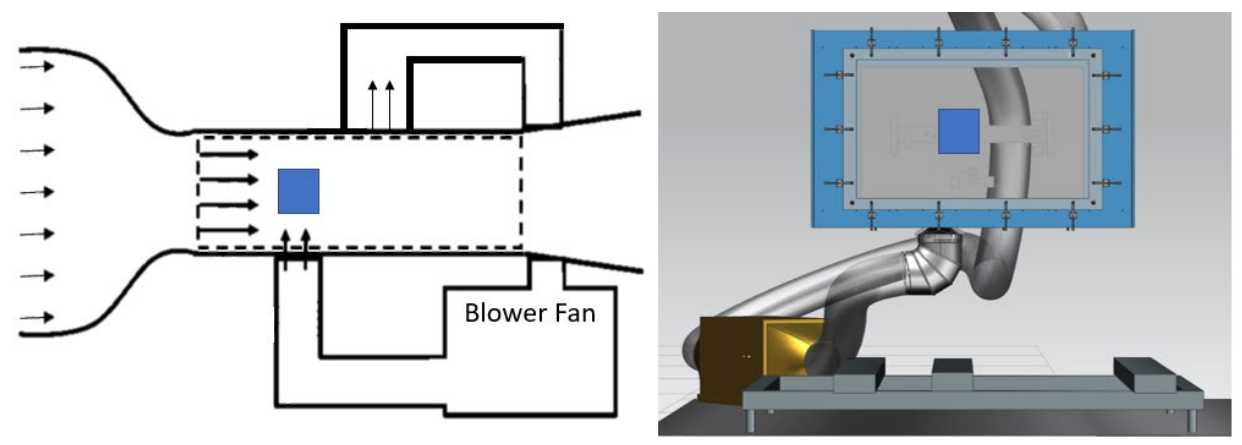

Fig. 1 Gust generator setup schematic and CAD drawing

PIV data was recorded using a LaVision Imager Pro X 4M $(2112 \times 2072$ pix $)$ camera and a Litron Nano PIV laser illumination $(532 \mathrm{~nm})$. The seed particles used were atomized Di-Ethyl-Hexyl-Sebacat (DEHS) oil droplets that spread evenly throughout the flow. A $60 \mathrm{~mm}$ Nikon lens was used, and the resulting field of view was approximately $32.5 \mathrm{x}$ $32.5 \mathrm{~cm}$, which covered roughly the middle-third of the test section, Fig. 2. Correlation was done using a multi-step correlation. A square 32x32 pixel window with no window overlap was used to pre-target a 24-pixel radius circular window with a $25 \%$ window overlap. Two passes were done with the smaller window and the process resulted in a 
vector spacing of approximately $3 \mathrm{~mm}$ between vectors. A time step $\Delta \mathrm{t}$ between images of $150 \mu \mathrm{s}$ was used for all tests.

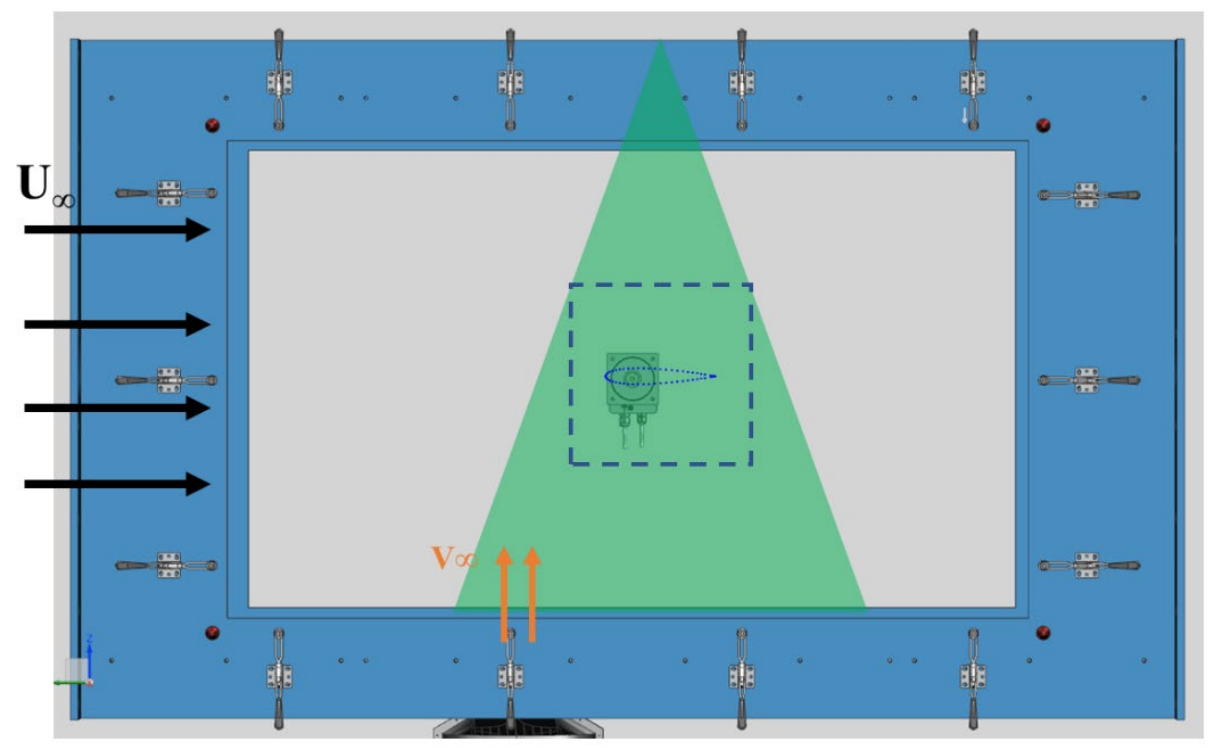

Fig. 2 PIV field of view

In addition to the steady state characterization, the PIV was phased locked to the opening of the fan slats of the gust fan. Time resolution was effectively increased by phase locking the PIV recording to gust initiation. Data resolution was increased from $7 \mathrm{~Hz}$ to $35 \mathrm{~Hz}$ by delaying the PIV trigger in relation to the gust initiation time. A set of 50 repeated trials were done at every phase and the vector fields from these repeated trials were remapped onto a regular grid based on the technique used in Cohn and Koochesfahani ${ }^{11}$.

\section{Numerical Methods}

\section{Governing Equations}

Unsteady compressible Navier-Stokes equations in conservation form are considered in the physical domain $(t, x, y, z)$ as follows:

$$
\frac{\partial Q}{\partial t}+\frac{\partial F}{\partial x}+\frac{\partial G}{\partial y}+\frac{\partial H}{\partial z}=0
$$

where $Q=(\rho, \rho u, \rho v, \rho w, E)^{T}$ are conservative variables, $\rho$ is the density of fluid, $, u, v$ and $w$ are the $\mathrm{x}-, \mathrm{y}-$ and $\mathrm{z}-$ components of the velocity and $E$ is the total energy given by $E=\frac{p}{\gamma-1}+\frac{1}{2} \rho\left(u^{2}+v^{2}+w^{2}\right)$ for a perfect gas in which $p$ is the pressure and $\gamma$ is the constant specific heat capacity ratio. The total energy equation closes the solution system. $F, G$ and $H$ are total flux vectors including the inviscid and viscous flux terms in the x-, y-, and z-direction, respectively. They are expressed as

$$
F=F^{i}-F^{v}=\left\{\begin{array}{c}
\rho u \\
\rho u^{2}+p-\tau_{x x} \\
\rho v u-\tau_{x y} \\
\rho w u-\tau_{x z} \\
u(E+p)-u \tau_{x x}-v \tau_{x y}-w \tau_{x z}-\frac{\mu C_{p}}{P r} T_{x}
\end{array}\right\}
$$




$$
\begin{aligned}
& G=G^{i}-G^{v}=\left\{\begin{array}{c}
\rho v \\
\rho u v-\tau_{y x} \\
\rho v^{2}+p-\tau_{y y} \\
\rho w v-\tau_{y z} \\
v(E+p)-u \tau_{y x}-v \tau_{y y}-w \tau_{y z}-\frac{\mu C_{p}}{P r} T_{y}
\end{array}\right\} \\
& H=H^{i}-H^{v}=\left\{\begin{array}{c}
\rho w \\
\rho u w-\tau_{z x} \\
\rho v w-\tau_{z y} \\
\rho w^{2}+p-\tau_{z z} \\
w(E+p)-u \tau_{z x}-v \tau_{z y}-w \tau_{z z}-\frac{\mu C_{p}}{P r} T_{z}
\end{array}\right\} \text {, }
\end{aligned}
$$

where $\mu$ is the dynamic viscosity, $C_{p}$ is the specific heat at constant pressure, $\operatorname{Pr}$ is the Prandtl number and $T$ is the temperature. For the Newtonian fluids, the viscous stresses are given as follows:

$$
\begin{array}{ll}
\tau_{x x}=2 \mu\left(u_{x}-\frac{u_{x}+v_{y}+w_{z}}{3}\right), & \tau_{y y}=2 \mu\left(v_{y}-\frac{u_{x}+v_{y}+w_{z}}{3}\right), \\
\tau_{z z}=2 \mu\left(w_{z}-\frac{u_{x}+v_{y}+w_{z}}{3}\right), & \tau_{x y}=\tau_{y x}=\mu\left(v_{x}+u_{y}\right), \\
\tau_{x z}=\tau_{z x}=\mu\left(w_{x}+u_{z}\right), & \tau_{y z}=\tau_{z y}=\mu\left(w_{y}+v_{z}\right) .
\end{array}
$$

The governing equation (1) in the physical domain $(t, x, y, z)$ is transformed to computational domain $(\tau, \xi, \eta, \zeta)$ as shown in Eq. (4). In the coordinate transformation, $\tau=t$ and $(\xi, \eta, \zeta) \in[-1,1] \times[-1,1] \times[-1,1]$ is a standard cubic element in the computational domain.

$$
\frac{\partial \tilde{Q}}{\partial \tau}+\frac{\partial \tilde{F}}{\partial \xi}+\frac{\partial \tilde{G}}{\partial \eta}+\frac{\partial \widetilde{H}}{\partial \zeta}=0
$$

where

$$
\left\{\begin{array}{c}
\tilde{Q}=|J| Q \\
\tilde{F}=|J|\left(Q \xi_{\tau}+F \xi_{x}+G \xi_{y}+H \xi_{z}\right) \\
\tilde{G}=|J|\left(Q \eta_{\tau}+F \eta_{x}+G \eta_{y}+H \eta_{z}\right) \\
\widetilde{H}=|J|\left(Q \zeta_{\tau}+F \zeta_{x}+G \zeta_{y}+H \zeta_{z}\right)
\end{array} .\right.
$$

During the coordinate transformation, the Jacobian matrix can be written as the following form:

$$
J=\frac{\partial(x, y, z, t)}{\partial(\xi, \eta, \zeta, \tau)}=\left(\begin{array}{cccc}
x_{\xi} & x_{\eta} & x_{\zeta} & x_{\tau} \\
y_{\xi} & y_{\eta} & y_{\zeta} & y_{\tau} \\
z_{\xi} & z_{\eta} & z_{\zeta} & z_{\tau} \\
0 & 0 & 0 & 1
\end{array}\right) .
$$

The inverse transformation must also exist for a non-singularity transformation, which can be related to the Jacobian matrix as:

$$
J^{-1}=\frac{\partial(\xi, \eta, \zeta, \tau)}{\partial(x, y, z, t)}=\left(\begin{array}{cccc}
\xi_{x} & \xi_{y} & \xi_{z} & \xi_{t} \\
\eta_{x} & \eta_{y} & \eta_{z} & \eta_{t} \\
\zeta_{x} & \zeta_{y} & \zeta_{z} & \zeta_{t} \\
0 & 0 & 0 & 1
\end{array}\right)
$$

\section{Spatial Discretization and Time Integration Methods}

A three dimensional (3D) SD method on dynamic unstructured grids developed in a series of works by Yu et al. ${ }^{12-}$ 15 is used to solve the governing equations. For completeness, the SD formulation in a standard hexahedral element is expressed as 


$$
\begin{aligned}
\frac{\partial \widetilde{Q}(\xi, \eta, \zeta)}{\partial \tau} & +\sum_{i=1}^{N+1} \sum_{j=1}^{N} \sum_{k=1}^{N}\left(\tilde{F}_{i, j, k}^{i n v, I}-\tilde{F}_{i, j, k}^{v i s, I}\right) \cdot \frac{d}{d \xi} l_{F P s, i}(\xi) \cdot l_{S P S, j}(\eta) \cdot l_{S P s, k}(\zeta) \\
& +\sum_{i=1}^{N} \sum_{j=1}^{N+1} \sum_{k=1}^{N}\left(\tilde{G}_{i, j, k}^{i n v, I}-\tilde{G}_{i, j, k}^{v i s, I}\right) \cdot l_{S P S, i}(\xi) \cdot \frac{d}{d \eta} l_{F P S, j}(\eta) \cdot l_{S P S, k}(\zeta) \\
& +\sum_{i=1}^{N} \sum_{j=1}^{N} \sum_{k=1}^{N+1}\left(\widetilde{H}_{i, j, k}^{i n v, I}-\tilde{G}_{i, j, k}^{v i s, I}\right) \cdot l_{S P s, i}(\xi) \cdot l_{S P s, j}(\eta) \cdot \frac{d}{d \zeta} l_{F P s, k}(\zeta)=0 .
\end{aligned}
$$

Note that two sets of points, namely solution points and flux points, are used in the SD method. In Eq. (8), $l_{F P S}$ stands for the flux-points-based Lagrange polynomial, $l_{S P S}$ stands for the solution-points-based Lagrange polynomial, and the superscript ' $I$ ' indicates that the fluxes are continuous across different elements. To ensure the flux continuity on element surfaces, the common inviscid fluxes are reconstructed with the $\mathrm{AUSM}^{+}$-up Riemann solver for all speeds ${ }^{16}$, and the common viscous fluxes are reconstructed with the 'BR1' approach developed in the Ref. 17.

The explicit three-stage strong stability preserving (SSP) Runge-Kutta method ${ }^{18}$ is used for time integration. For any ordinary differential equation in the form $d Q / d t=R(Q)$, the three-stage SSP Runge-Kutta method is given by

$$
\left\{\begin{array}{c}
Q^{(1)}=Q^{n}+\Delta t R\left(Q^{n}\right) \\
Q^{(2)}=\frac{3}{4} Q^{n}+\frac{1}{4} Q^{(1)}+\frac{1}{4} \Delta t R\left(Q^{(1)}\right) . \\
Q^{n+1}=\frac{1}{3} Q^{n}+\frac{2}{3} Q^{(2)}+\frac{2}{3} \Delta t R\left(Q^{(2)}\right)
\end{array}\right.
$$

\section{Computational Domain}

Figures 3 and 4 show the computational domain used for the numerical study. The dimensions of the computational domain were calculated to match the wind tunnel test section of the physical experiment. For these simulations, the fixed inlet and outlet boundary and the symmetry boundary condition on the bottom and the top of computational domain were implemented. For the gust inlet and outlet, the Gaussian profile velocity was implemented as the boundary condition. For this study, the gust velocity is only in vertical direction and is given as $V_{g}=\left(0, v_{g}, 0\right)$, where $v_{g}=2.39 * e^{-\left(\frac{x-\bar{x}}{51.22}\right)^{2}}$ measured experimentally ${ }^{6}$. In the simulation for the airfoil at Reynolds number 1,000 and 12,000 , the gust was turned on at the start of the simulation. For the simulation at Reynolds number 1,000, the computational domain shown in Fig. 3 was used. The computational domain shown in Fig. 4 was used for the simulation at Reynolds number 12,000 and fixed boundary on the top of computational domain was implemented. 


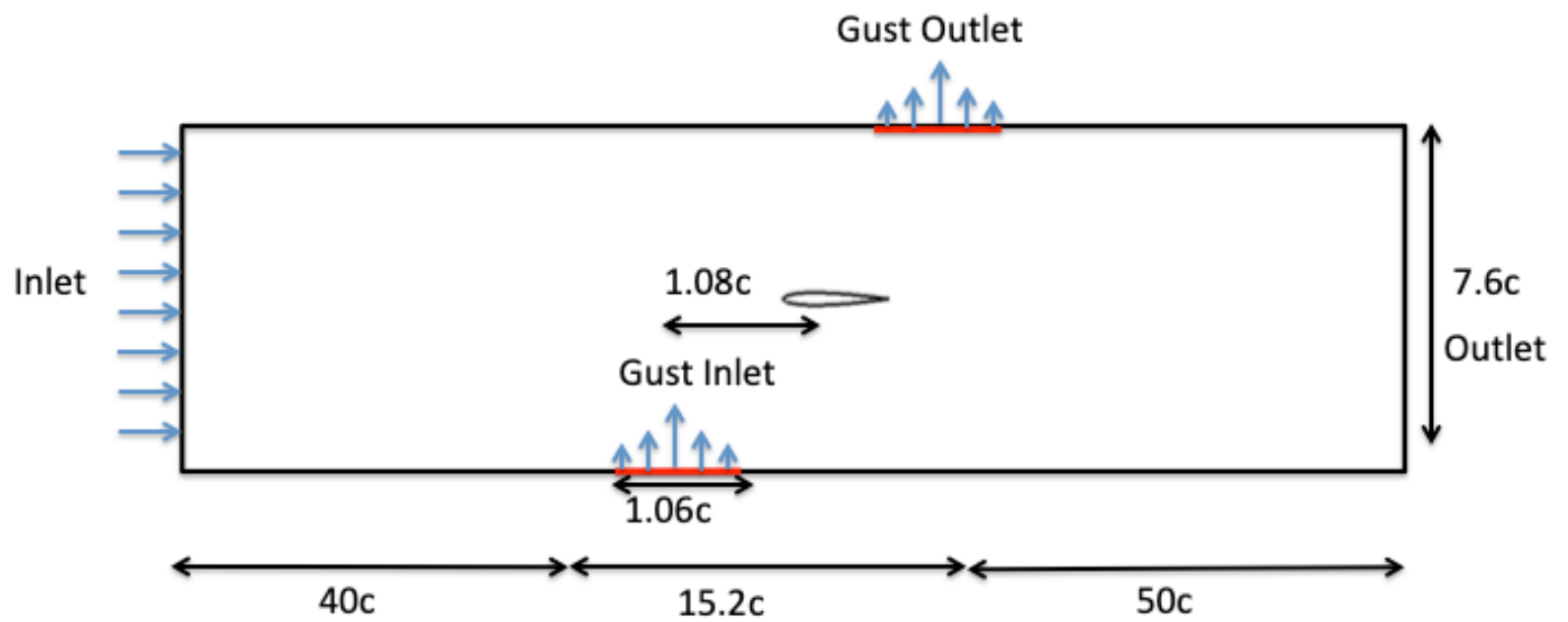

Fig. 3 Illustration of the computational domain

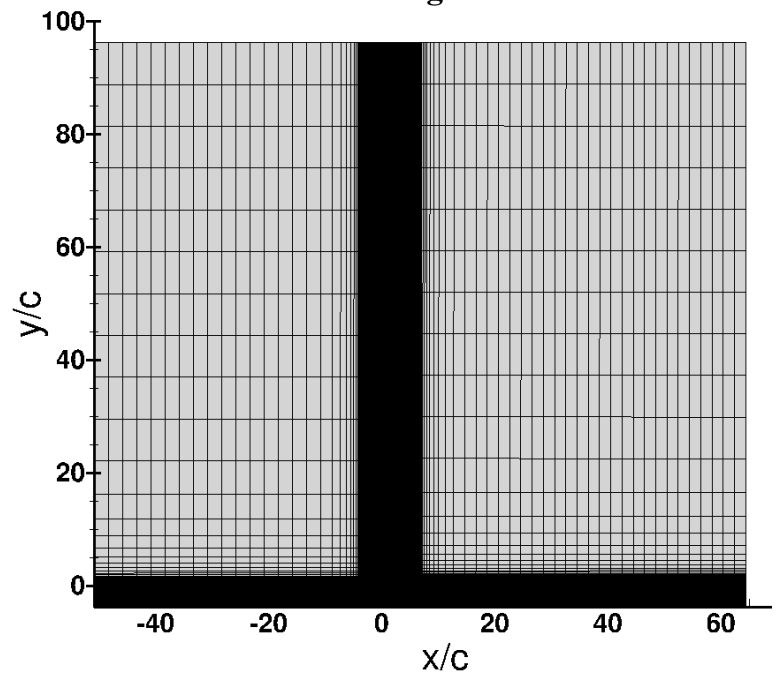

(a)

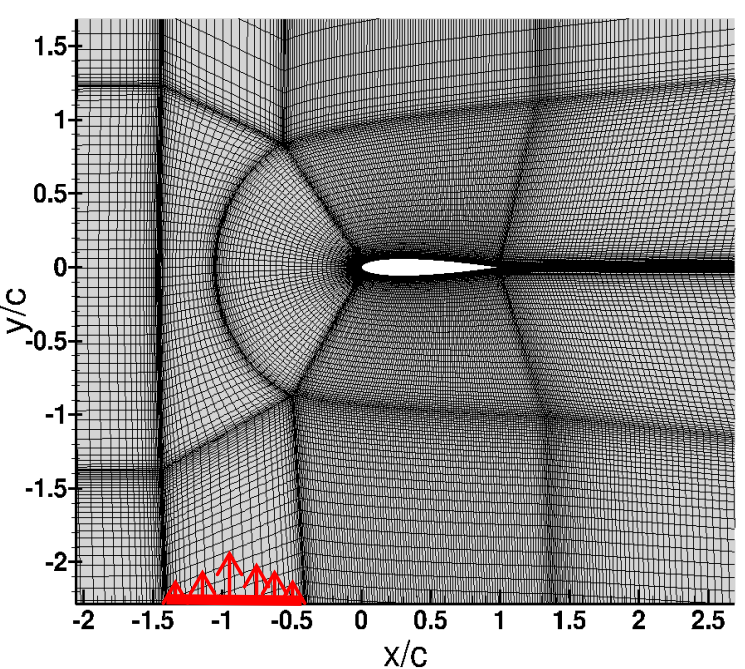

(b)

Fig. 4 (a) Computational mesh. (b) Close view of the mesh near the airfoil

\section{Results and Discussions}

Flow characterization results from Smith ${ }^{6}$ showed that the jet from the gust inlet bent in the freestream direction, effectively causing a blockage effect, which accelerated the flow upward. This effect generated a gust magnitude, $\mathrm{V} / \mathrm{U}_{\infty}$, of greater than $35 \%$. Some leakage from the gust design limited the effectiveness of actuating the gust to a change in flow angle of approximately $15^{\circ}$, but this was more than significant enough to cause a stall event. Figure 5 shows the evolution of the gust properties at two separate values of $t^{*}$, where $t^{*}=\left(t-t_{\text {gust }}\right) * U_{\infty} / c$. Around $t *=5.36$ the gust was still developing in the test section while at $t^{*}=26.79$ the gust was near-fully developed. 


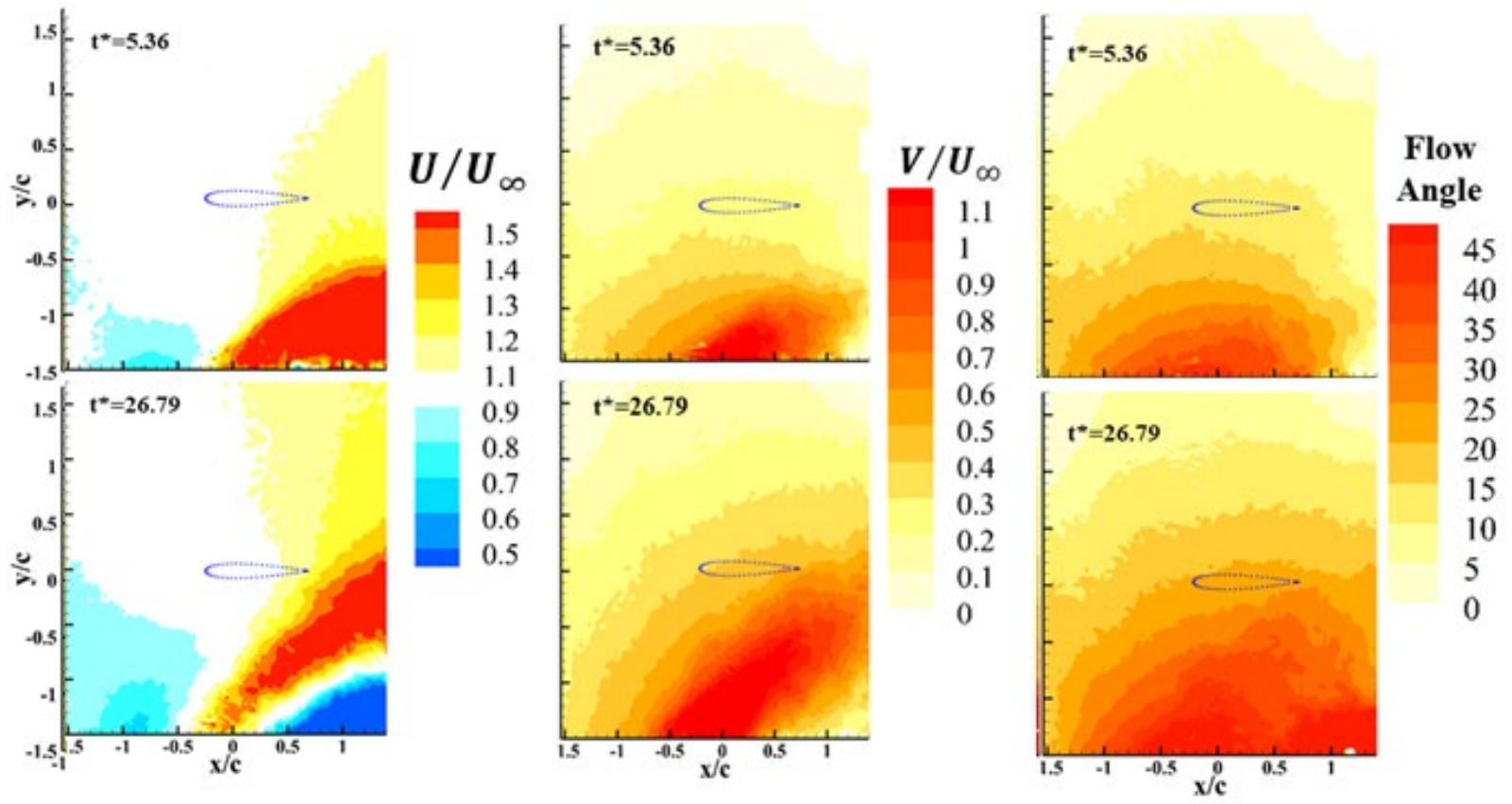

Fig. 5 Gust development in the PIV experiment.

The results showed that the gust minimally altered the free stream velocity near the planned test area, highlighted by the dashed airfoil although no wing was present during this test. However, the vertical velocity of the flow and flow angle grew significantly. Interestingly the flow angle grew in a somewhat uniform manner over the chord of the wing. The flow angle in that region was extracted and is shown in Fig. 6, which includes raw data points and a smoothing fit of local points.

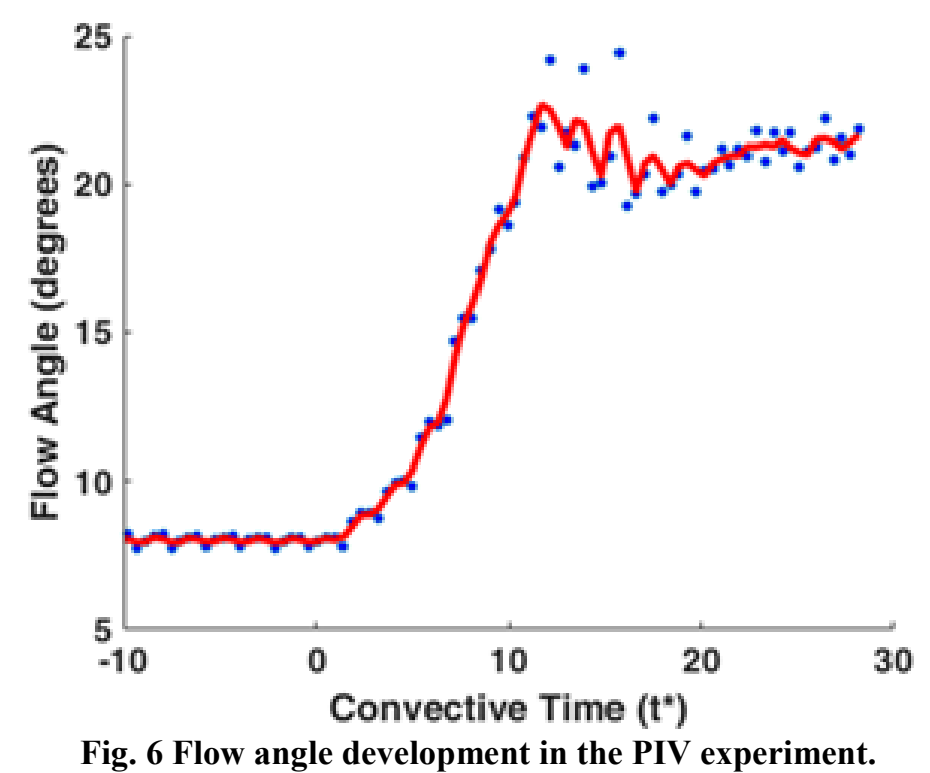

The results showed that there was a leakage of flow in the test section from the gust generator, resulting in a nonzero flow angle prior to the actuation of the gust. While flow angle corrections were made for the study by Smith, the study conducted there leaves open the question of exactly how best to improve the design of the gust generator. Ideally, CFD results capable of matching the results in Smith will be capable of advising the redesign while the gust results inform improvements and understanding in the CFD methods that need to be used in gust interaction problems. 
The results shown in Fig. 7 are the computational gust development at $t^{*}=5.32$ (Fig. 7 (a) and (b)) and $t^{*}=26.76$ (Fig. 7 (c) and (d)). Fig. 7 (b) shows that the gust was developing by bending the gust velocity profile in the freestream direction when interacting with the freestream. At $t^{*}=26.76$ the gust near the planned test area is fully developed as shown in Fig. 7 (d). Computational and experimental results in Figs. 5 and 7 have the similar profile of velocity and have the similar values. There is, however some difference in magnitude of maximum values for the normalized horizontal $\frac{U}{U_{\infty}}$ and vertical $\frac{V}{U_{\infty}}$ velocities. Also, when closely compared there were some differences in the convective time. Comparing CFD results with the experimental at the planned test section, it has the similar behavior of the gust growth over time. The blue dotted box is the position where the airfoil is supposed to be placed for gust wing interaction.
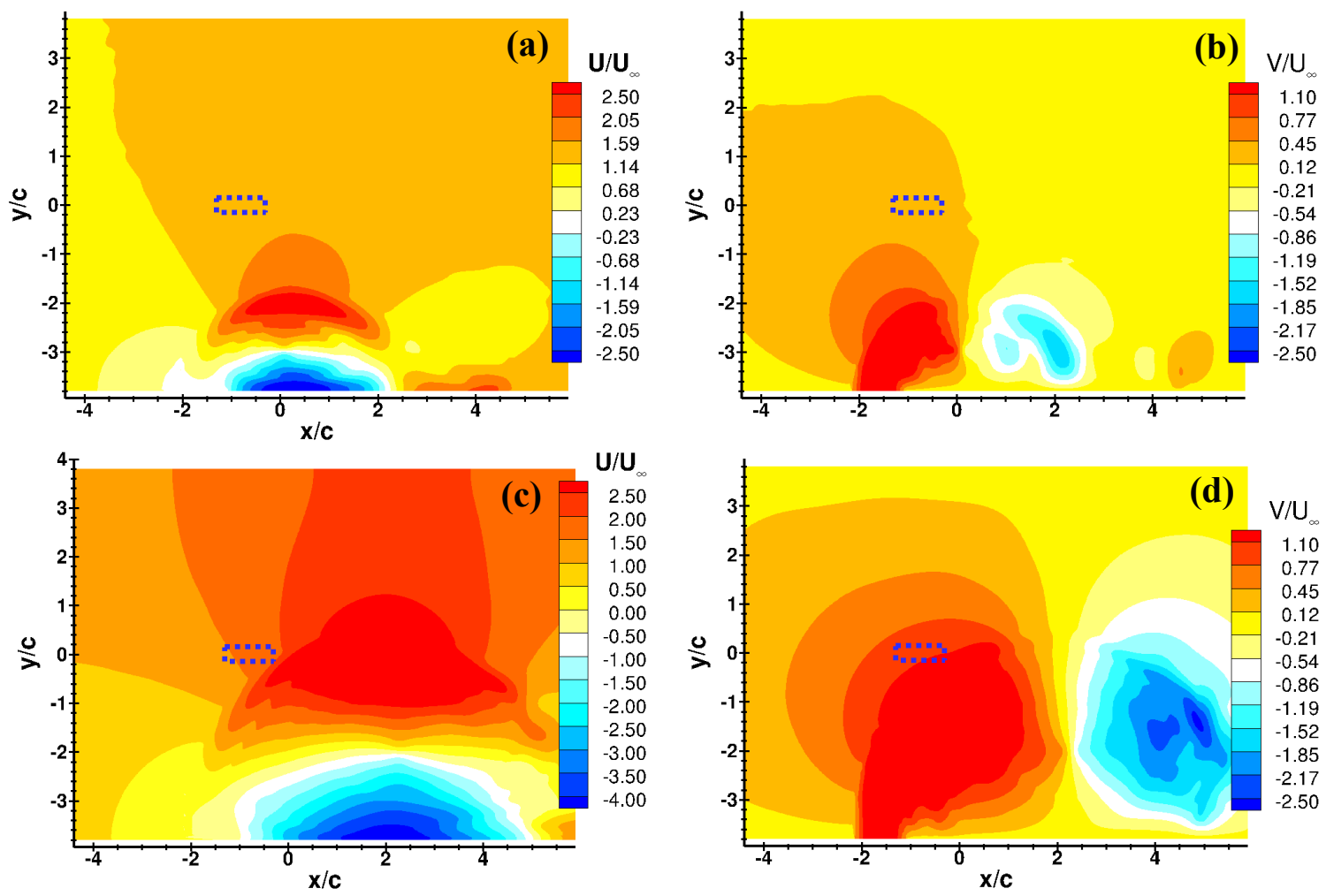

Fig. 7 Computational gust development (a) $\frac{U}{U_{\infty}}$ at $t^{*}=5.32$, (b) $\frac{V}{U_{\infty}}$ at $t^{*}=5.32$, (c) $\frac{U}{U_{\infty}}$ at $\mathrm{t}^{*}=26.76$ and (d) $\frac{V}{U_{\infty}}$ at $\mathrm{t}^{*}=26.76$.

\section{Gust Wing Interaction at 0 degree Angle of Attack and Reynolds Number 1,000}

To rapidly understand whether the behavior of the gust generator could be simulated, a fast computation was run at lower Reynolds number in search of similar flow behaviors. In this section, the computational flow features observed during the vertical gust interacting with the airfoil at Reynolds number 1,000 are discussed. As the vertical gust grows, it started to interact with the wing at non-dimensional time $t^{*}=9.08$. As the gust was interacting with airfoil, the shear layer from the airfoil began to bend towards the leading edge of the suction side of the airfoil shown in Fig. 8 (a). As the interaction progressed to non-dimensional time $t^{*}=14.70$, the separation bubble grew causing the flow to separate from the trailing edge of the airfoil, Fig. 8 (b) and (c). When the gust reached the non-dimensional time of $\mathrm{t}^{*}=26.72$, shown in Fig. 8(d), flow separation was observed on the top surface of the airfoil the airfoil entered an unsteady stalled condition. The same flow phenomena were observed in the experiment at Reynolds number 12,000 , suggesting that the gust generator behavior was somewhat Reynolds number independent in the Reynolds number range tested in this study. 

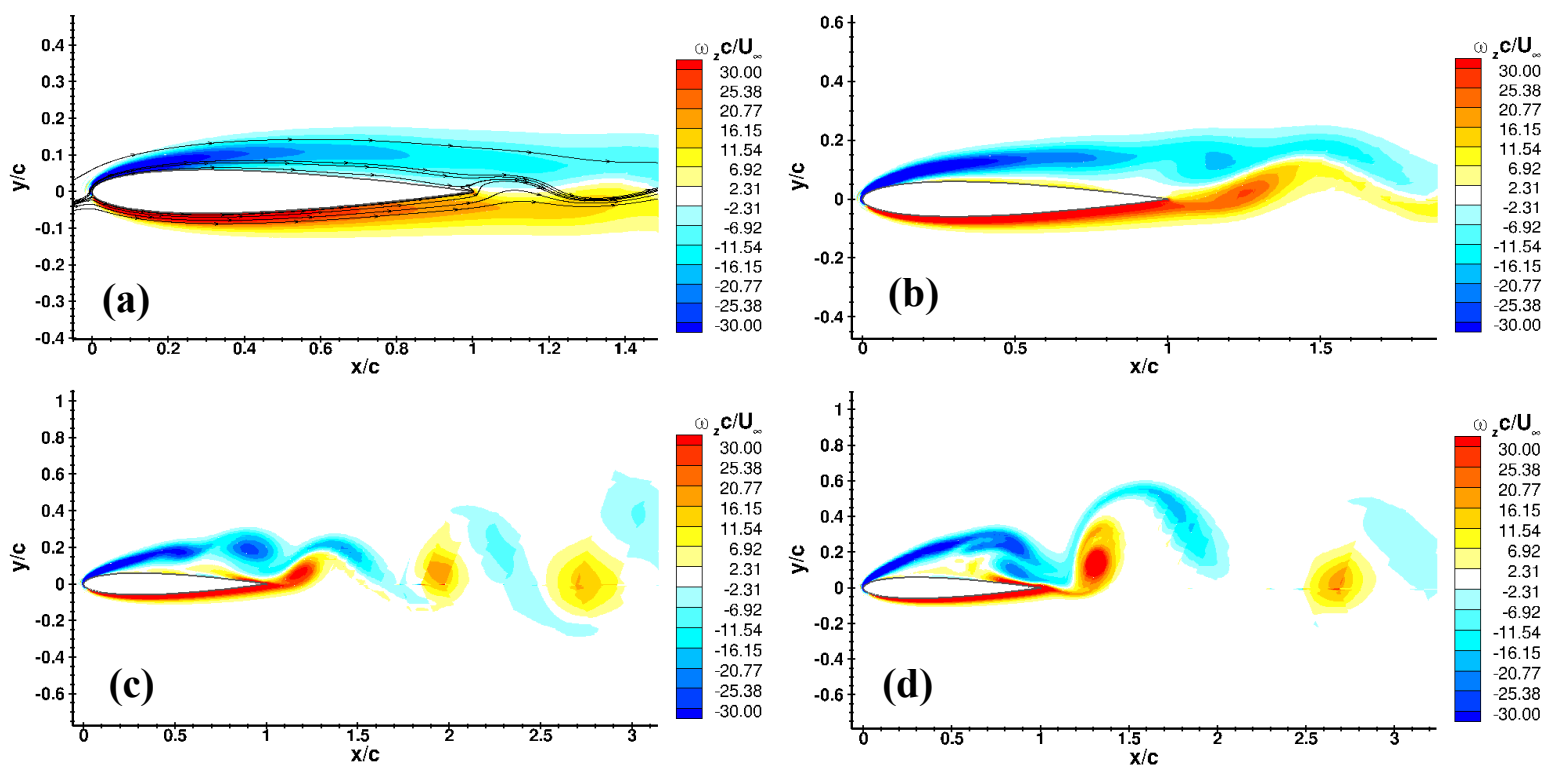

Fig. 8 Instantaneous vorticity fields at (a) $t^{*}=9.08,(b) t^{*}=14.70$,

(c) $t^{*}=20.04$ and (d) $t^{*}=26.72$ at $\operatorname{Re}=1,000$.

\section{Gust-Wing-Interaction at 0 degree Angle of Attack and Reynolds Number 12,000}

Once the gust effects were shown to be replicable, the gust was simulated at Reynolds number and inflow conditions that closely matched the experiments. Due to experimental limitations, an exact match for the gust inflow conditions was not possible but all other flow conditions were matched. The effect of gust on the bottom boundary layers of the airfoil can be seen as early as non-dimensional time $t^{*}=1.105$ and start to form the separation bubble as shown in Fig. 9 (b). The separation bubble grew in time and the separation started to occur near the trailing edge of the airfoil shown in Fig. 10. After $t^{*}=5.35$ the upper surface shear layer began to separate and advanced toward leading edge due to the gust interaction as shown in Fig. 11. After $t^{*}=5.35$, as the shear layer from the bottom surface of the airfoil passes toward the leading edge of the top surface of the airfoil, the vortices start to form from the top surface and detached from the airfoil forming the vortex shedding as shown in Fig. 12.

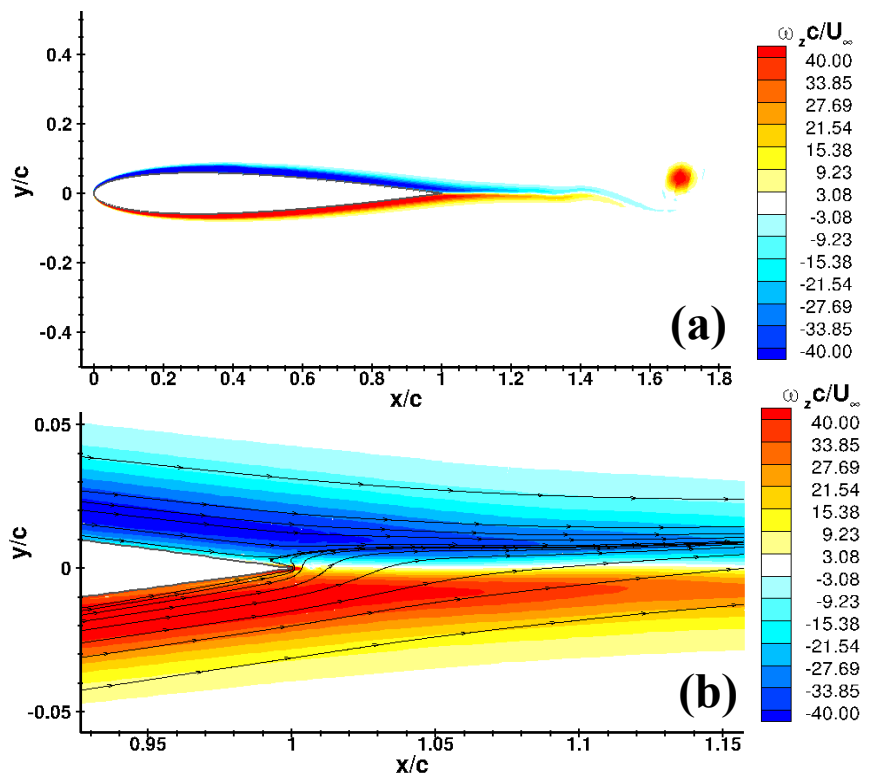

Fig. 9 (a) An instantaneous vorticity field at $t^{*}=1.105$ and

(b) Close view of separation bubble at the tailing edge of airfoil at $\operatorname{Re}=12,000$. 

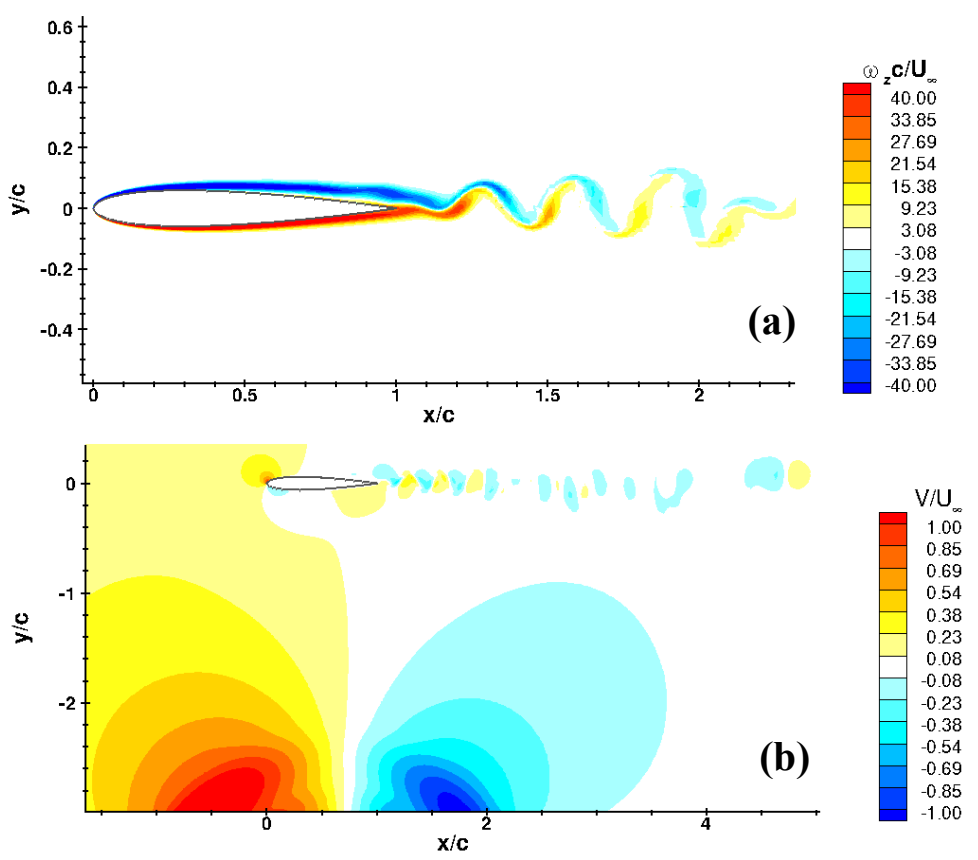

Fig. 10 (a) An instantaneous vorticity field at $t^{*}=3.99$ and (b) $\frac{V}{U_{\infty}}$ at $t^{*}=3.99$ at $\operatorname{Re}=12,000$.
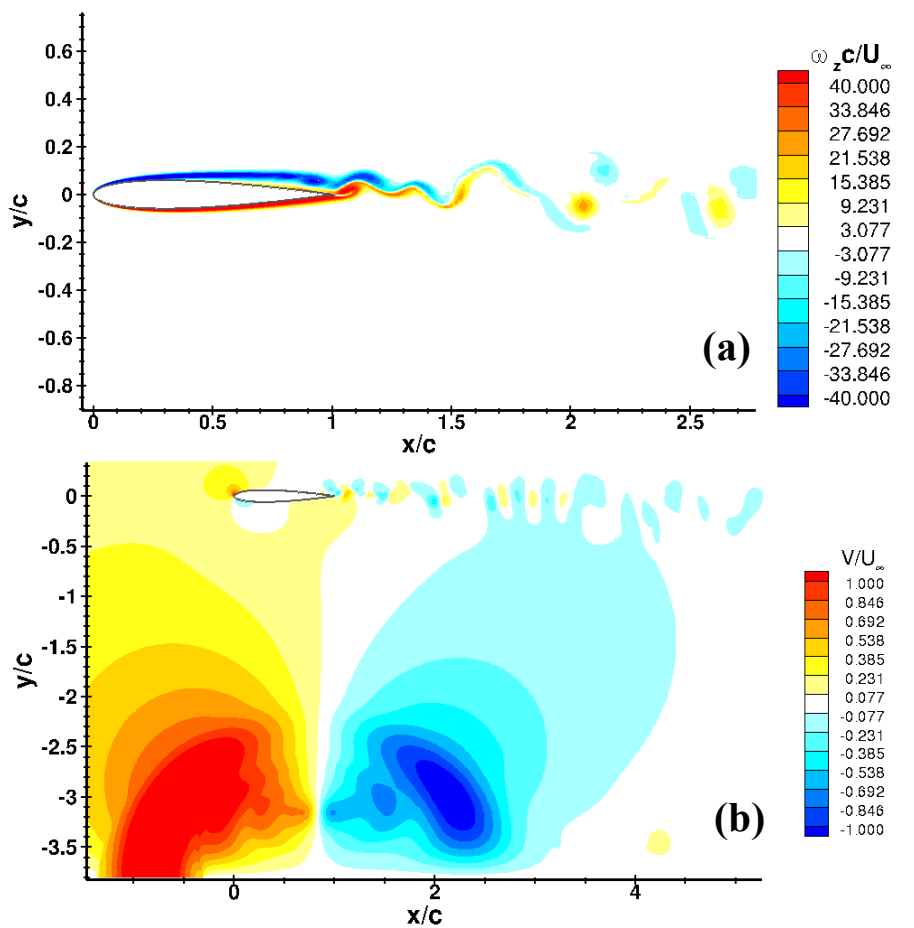

Fig. 11 (a) An instantaneous vorticity field at $t^{*}=5.35$ and (b) $\frac{V}{U_{\infty}}$ at $t^{*}=5.35$ at $\operatorname{Re}=12,000$. 


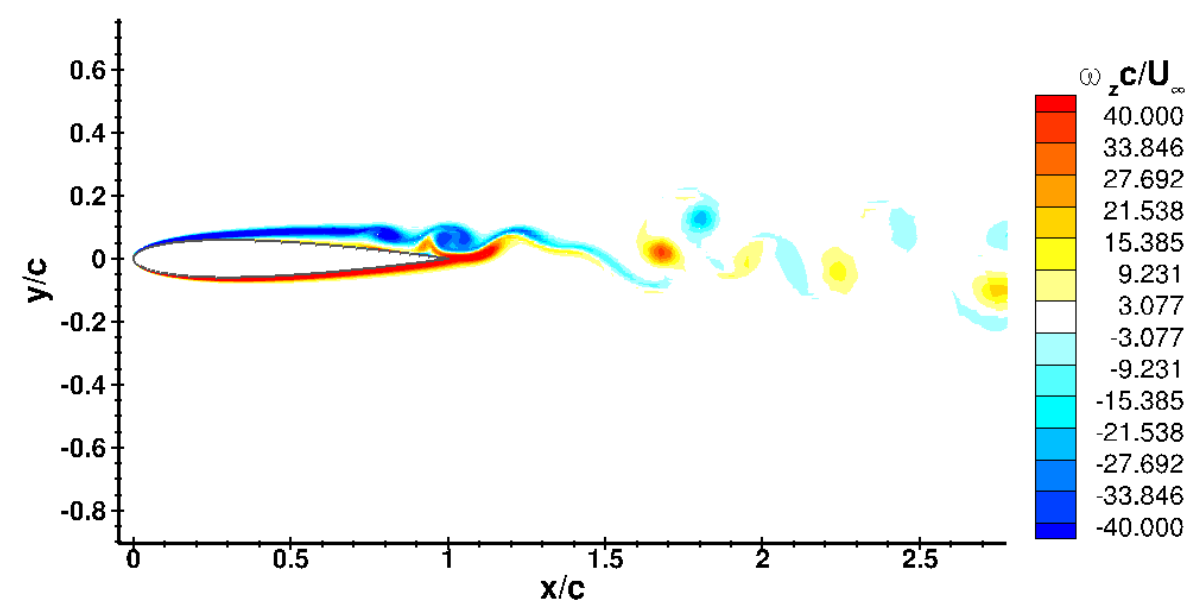

Fig. 12 An instantaneous vorticity field at $t^{*}=5.99$ at $\operatorname{Re}=12,000$.

\section{Comparison of Experimental and Computational Results}

Figures 13 and 14 show the computational and experimental contours of vorticity for the NACA0012 airfoil interacting with the gust development over time. The effect of gust on the airfoil was observed after $t^{*}=5.36$ in the experiment while in the computational study this effect occurred after $t^{*}=1.105$. In the experimental results, the separation bubble was formed around $t^{*}=10.71$ non-dimensional time but in the computational results these phenomena were also observed early, around $t^{*}=1.105$. These discrepancies between the experimental and computational results may be due to the leakage of flow in the vertical gust in the tunnel in experiment and the corrections made for them. Also, increases in freestream turbulence levels, not present in the computation but inherently introduced by the gust generator in the wind tunnel, could have delayed flow separation in the experiments.

One interesting result was that the experimental flow field more closely resembled the lower Reynolds number case tested. Recent testing in the MAWT facility has shown that at low Reynolds numbers, freestream turbulence delayed the static stall of a NACA 0012. This comparison between experiments and computation suggest that a significant reduction in turbulence levels is critical to any gust generator in a wind tunnel. 


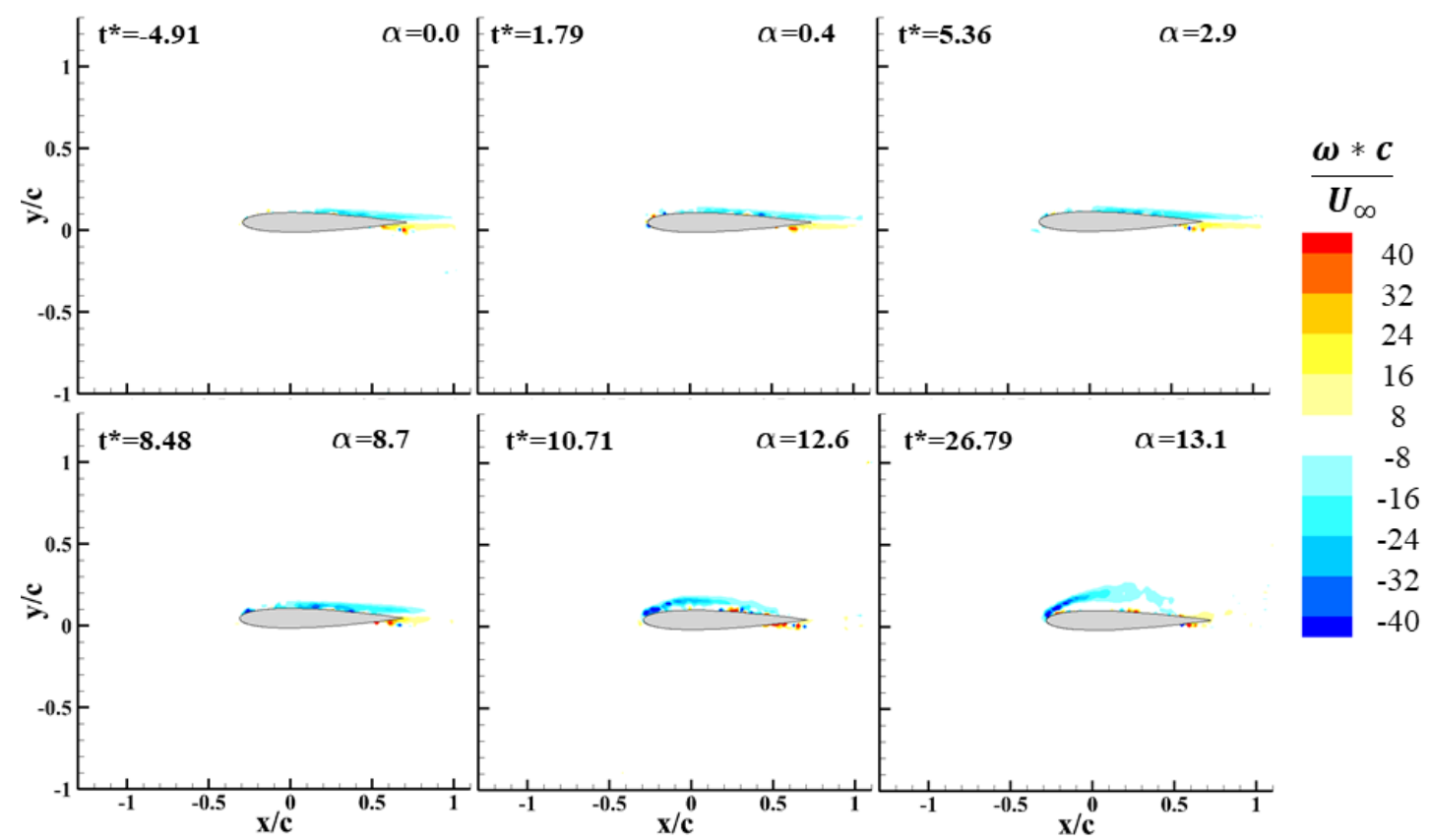

Fig. 13 Experimental gust wing interaction at 0 degree AoA.
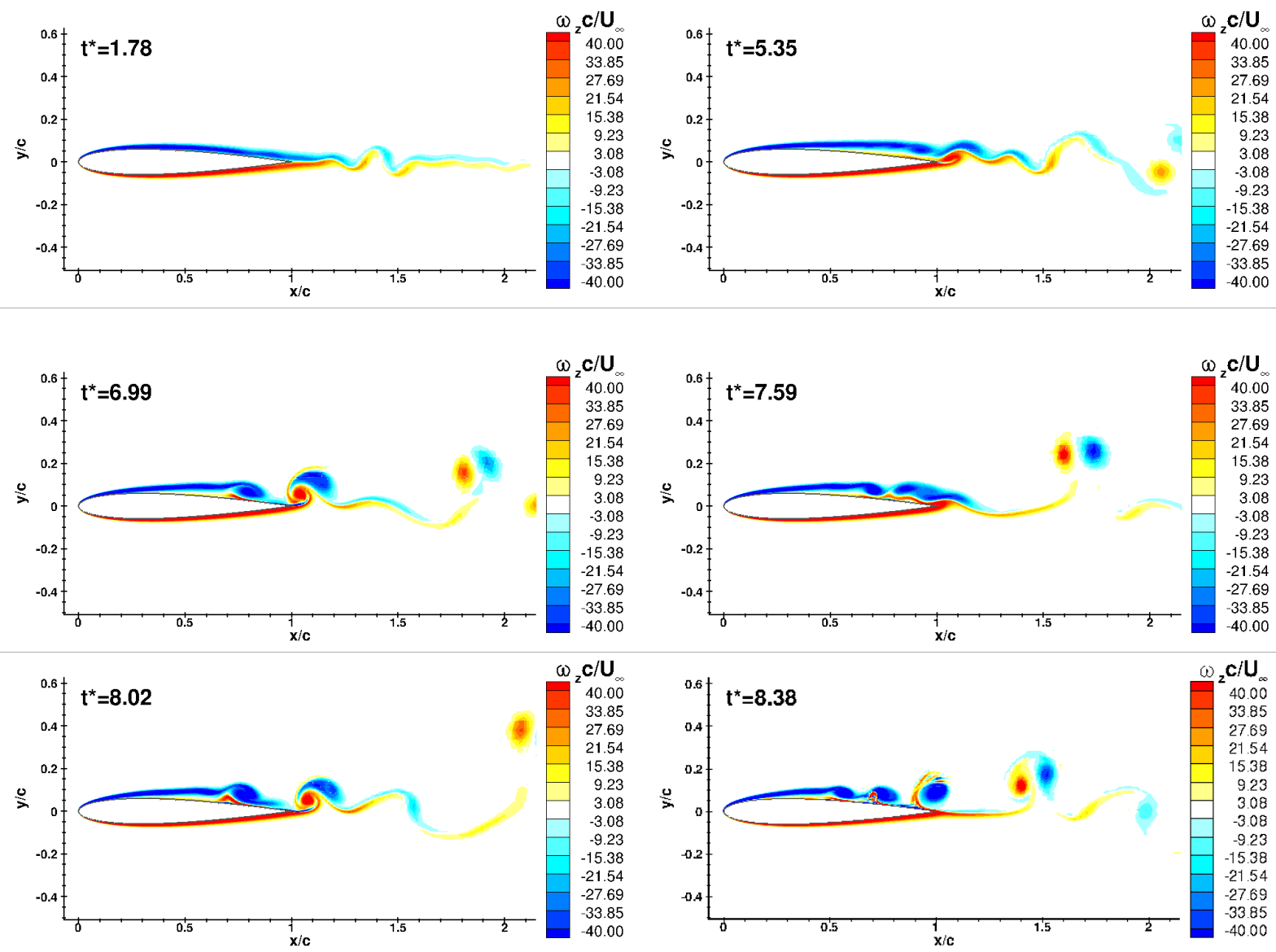

Fig. 14 Computational gust wing interaction at 0 degree AoA. 


\section{Conclusion}

Experimental and computational studies have been performed to investigate the flow phenomena over a NACA0012 airfoil when interacting with a vertical gust in a wind tunnel environment. During the gust development time, the effective angle of attack over the airfoil was rapidly changed causing the wing to undergo stall. The highorder spectral difference method was able to capture the unsteady wakes during the gust wing interaction at Reynolds numbers 1,000 and 12,000. Different fine-scale flow separation structures can be observed at the two Reynolds numbers. The numerical results agree reasonably well with the experimental results on revealing the stall process, but critical difference in the flow fields indicate that unknown factors are affecting the flow behavior. The most likely source of difference between the computation and the experimental results was freestream turbulence generated by the gust generator caused by flow leaking into the wind tunnel. Future work will include the continuation of gust wing interaction at different angle of attacks for stationary wing and the gust wing interaction on flapping wing. Furthermore, the parameter variables like gust ratios and gust positions will be explored computationally in order to better design the vertical gust generator in the wind tunnel.

\section{Acknowledgements}

Research was partially sponsored by the Army Research Laboratory and was accomplished under Cooperative Agreement Number W9911NF-16-2-0176. The views and conclusions in this document are those of the authors and should not be interpreted as representing the official policies, either expressed or implied, of the Army Research Laboratory or the U.S. Government. The U.S. Government is authorized to reproduce and distribute reprints for Government purposes notwithstanding any copyright notation herein.

\section{References}

[1] Donely, P., Summary of Information Relating to Gust Loads on Airplanes, NACA Report 997, 1950.

[2] Xu, X.P., Zhu, X.P., Zhou, Z., Chang, M., Numerical Simulation of Gust Response for UAV Airfoil, Proceedings of the 2010 Asia-Pacific International Symposium on Aerospace Technology, Vol 1 and 2, pp. 403, 2010

[3] Liauzun, C., Aeroelastic Response to Gust Using CFD Techniques, Proceedings of the ASME Fluids Engineering Summer Meeting, pp. 269-276, 2010

[4] An, J.G., Yan, Z., Qiu, C.R., Zhou, W.B., The Numerical Computation of Aircraft Response to Arbitrary Vertical Gust Distributions, Journal of Aircraft, Vol. 22 (11), pp. 988-992, 1985

[5] Smith, Z.F., Jones, A.R., Hrynuk, J.T., Micro Air Vehicle Scale Gust-Wing Interaction in a Wind Tunnel, 2018 AIAA Aerospace Sciences Meeting, AIAA SciTech Forum, 2018-0573

[6] Smith, Z.F., Micro Air Vehicle Scale Gust-Wing Interaction in a Wind Tunnel, Master of Science Thesis, University of Maryland, College Park, MD, 2018

[7] Patel, M. H., Hancock, G. J., A gust tunnel facility, Aeronautical Research Council Reports and Memoranda, Reports and memoranda number 3802, June 1976.

[8] Buell, D. A., An experimental investigation of the velocity fluctuations behind oscillating vanes, NASA Technical note NASA TN D-5543, Nov. 1969.

[9] Baik, Y., Bernal L., Granlund K., Ol M., Unsteady force generation and vortex dynamics of pitching and plunging aerofoils, Journal of Fluid Mechanics, Vol. 709, Aug. 2012.

[10] Perrotta G., Jones A., Unsteady forcing on a flat-plate wing in large transverse gusts, Experiments in Fluids 58:101, Aug. 2017.

[11] Cohn, R. K., Koochesfahani, M. M., The accuracy of remapping irregularly spaced velocity data onto a regular grid and the computation of vorticity, Experiments in Fluids, Suppl., S61-S69, 2000.

[12] Yu, M.L., Wang, Z.J., and Hu, H., A high-order spectral difference method for unstructured dynamic grids, Computers \& Fluids, vol. 48, Sep. 2011, pp. 84-97.

[13] Yu, M.L., Hu, H., and Wang, Z.J., Experimental and Numerical Investigations on the Asymmetric Wake Vortex Structures of an Oscillating Airfoil, AIAA-2012-0299, the 50th Aerospace Sciences Meeting Including the New Horizons Forum and Aerospace Exposition, Nashville, Tennessee, Jan. 9-12, 2012.

[14] Yu, M.L., Wang, Z.J., and Hu, H., High fidelity numerical simulation of airfoil thickness and kinematics effects on flapping airfoil propulsion, Journal of Fluids and Structures, Vol. 42, pp. 166-186, 2013.

[15] Yu, M.L., Wang, Z.J., and Hu, H., Formation of bifurcated wakes behind finite-span flapping wings, AIAA Journal, Vol. 51, No. 8, pp. 2040-2044,2013.

[16] M.-S. Liou, A sequel to AUSM, Part 2: AUSM+-up for all speeds, Journal of Computational Physics, vol. 214, 2006, pp. 137170. 
[17] F. Bassi, and S. Rebay, A high-order accurate discontinuous finite element method for the numerical solution of the compressible Navier-Stokes equations, Journal of Computational Physics, vol. 131, 1997, pp. 267-279.

[18] S. Gottlieb, C.-W. Shu, and E. Tadmor, Strong stability-preserving high-order time discretization methods, SIAM Rev., vol. 43, 2001, pp. 89-112. 